\title{
teoria del hormigón armado tomando en consideración el esfuerzo cortante
}

\author{
JOSE ANTONIO LOPEZ JAMAR Y JOSE JOAQUIN TIRADO CRUZ \\ Doctores Ingenieros de Caminos, Canales y Puertos
}

Los autores de este trabajo han finalizado su puesta a punto, lo cual representa un importante avance en el conocimiento del hormigón armado. Dado el interés que sin duda tendrá para muchos de nuestros lectores, hemos creído conveniente incluir en este número el resumen que sigue a continuación, avance de la monografía que se publicará en breve.

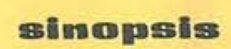

$450-8$

Este artículo es un resumen, anticipo de la teoría original de los señores López Jamar y Tirado Cruz, sobre flexión compuesta de piezas de hormigón armado, tomando en consideración el esfuerzo cortante.

La pieza se asimila a una viga en celosía; la cabeza superior, constituida por la faja sin fisurar, trabaja a compresión excéntrica con esfuerzo cortante, lo mismo que las diagonales de hormigón entre fisuras. L.a cabeza inferior, constituida por la armadura longitudinal, $y$ la otra serie de racción pura.

Partiendo de una ley parabólica de tensiones normales longitudinales, se obtienen rigurosamente le una ley parabólica de las tensiones de los estribos, se hallan las leyes de tensiones eeficaces» del hormigón de la zona fisurada a lo largo de la sección transversal recta.

Estas leyes de tensiones permiten estudiar las resistencias parciales de las fajas fisuradas y $\sin$ fisurar, esta última mediante la resistencia intrínseca. Resulta así que el esfuerzo cortante es función principalmente de la deformación unitaria horizontal relativa de la flbra o nivel que al. canzan las fisuras.

Del estudio se deducen cuatro posibles formas de agotamiento: transversal simultáneo del hormigón y estribos, armadura longitudinal, fibra más comprimida y agotamiento superior de la pieza. El complicado sistema de ecuaciones resultante se resuelve electrónicamente, obteniéndose listados mera comprobación mediante ensayos.

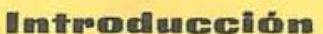

El estudio comienza por un rápido repaso crítico de las teorías y normas conocidas hasta la fecha, señalando los defectos generales de aquéllas y destacando la gran diversidad de criterios y resultados a que se llega con ellas. En la figura 1 se incluye un gráfico, muy expresivo por cierto, en el que se observan diferencias de hasta el 100 por 100 entre los resultados de algunas teorías.

En el estudio se consideran a continuación los tres estados posibles de una pieza prismática de hormigón solicitada a flexión compuesta y esfuerzo cortante (fig. 2): I, sin fisurar; II, fisurada en las zonas de flexión dominante; III, fisurada en la zona de inflexión.

La pieza se asimila a una viga en celosía con una cabeza de hormigón no fisurado sometida a compresión excéntrica con esfuerzo cortante; una cabeza extendida (armadura longitudinal); una serie de diagonales de tracción, o estribos (que eventualmente pueden no existir); y, finalmente, unas diagonales o bielas de hormigón entre fsuras, sometidas también a compresión excéntrica con esfuerzo cortante.

En la zona de inflexión el hormigón comienza su fsuración por dos puntos, situados algo al interior de las armaduras longitudinales y hacia las fajas extendidas, quedando en el centro una faja más o menos comprimida que constituye el enlace entre las fajas no fisuradas de las zonas de flexión dominante. 

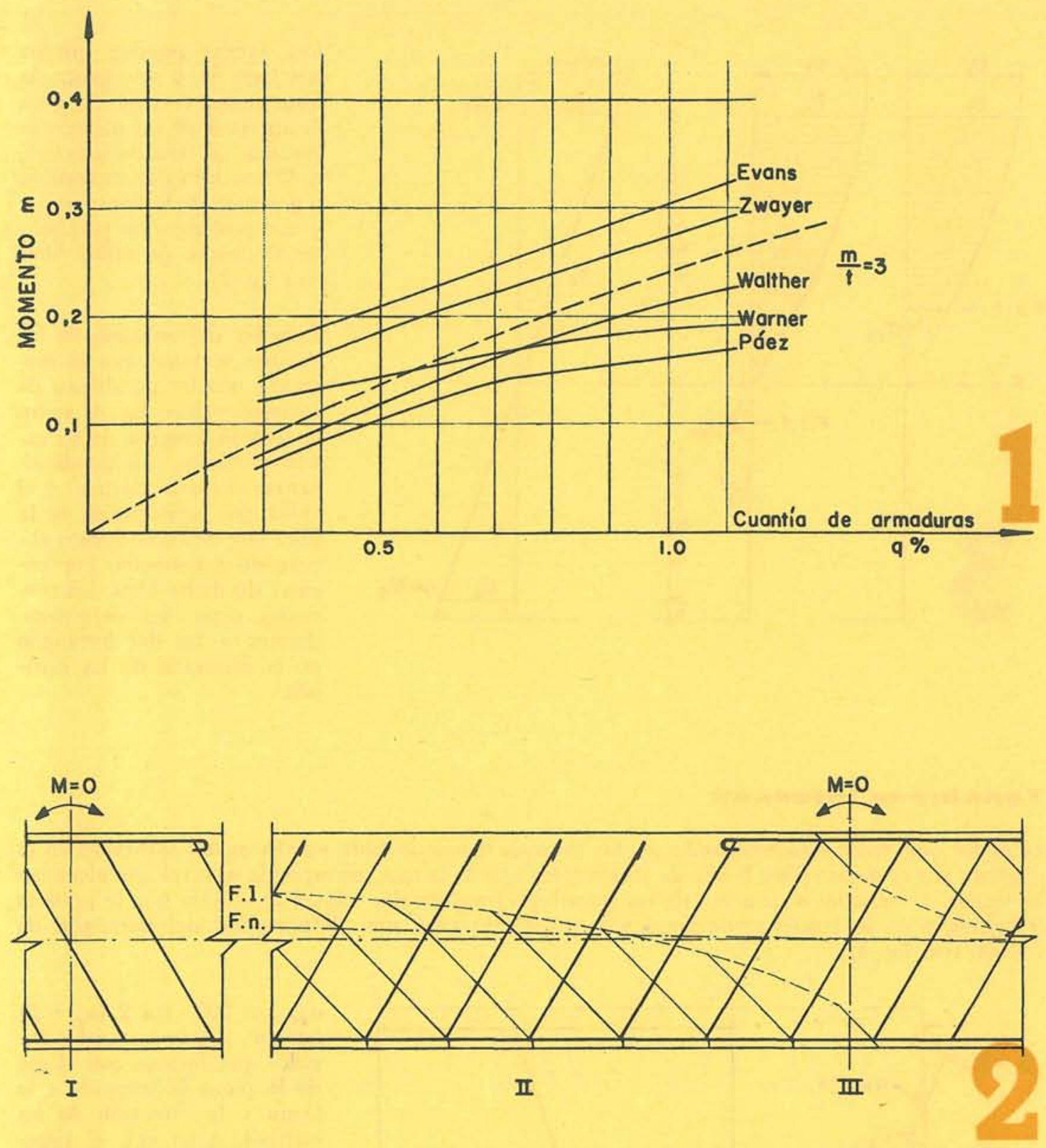

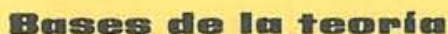

Se admite la hipótesis de deformación plana y se consideran, por el momento, leyes de tensiones-deformaciones parabólicas en el hormigón, de segundo y cuarto grado para compresiones y tracciones, respectivamente (fig. 3).

El estudio se concreta al caso de esfuerzo cortante constante (más desfavorable para cargas sobre la viga). Por ello se admite, para las zonas de flexión dominante, que las magnitudes siguientes tengan variación lenta con la abscisa: el esfuerzo axil; la cuantía, tensión e inclinación de las armaduras transversales; y la posición en profundidad de las diferentes resultantes parciales de las tensiones internas. 


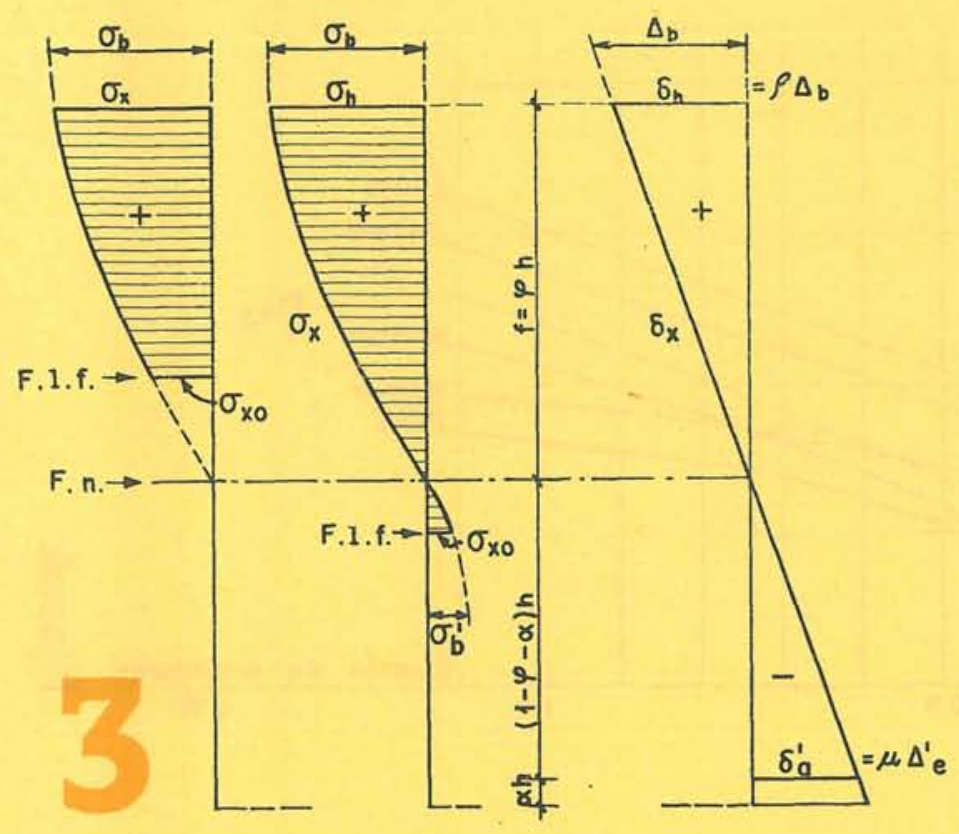

Las fisuras pueden quedar por bajo de, o sobrepasar, la fibra neutra (ver fig. 3). Para la apertura de las mismas se justifica un trazado práctico, a $45^{\circ}$ en las fajas extendidas o por bajo de la fibra neutra, y con prolongación parabólica si pasara de dicha fibra (ver fig. 2).

Para ley de tensiones en los estribos se toma, por el momento, una ley parabólica de segundo grado (fig. 4) entre la tensión máxima de su extremo inferior (que puede alcanzar el límite elástico) y el valor que corresponda en la fibra que denominaremos «límite de fisuración». Por encima de dicha fibra, las tensiones serán las correspondientes a las del hormigón en la dirección de los estribos.

\section{Temsionnes întremmens}

El papel que viene desempeñando en las diversas teorias la fibra neutra queda sustituido en la presente por el de la «fibra limite de fisuración», que es la que corresponde al nivel que alcanzan las fisuras. A dicho nivel, el acero de las armaduras transversales tomará la tensión que le permita el hormigón en su comienzo de fisuración, resultando para una deformación del hormigón de $1 / 3.000$ (ver fig. 4):

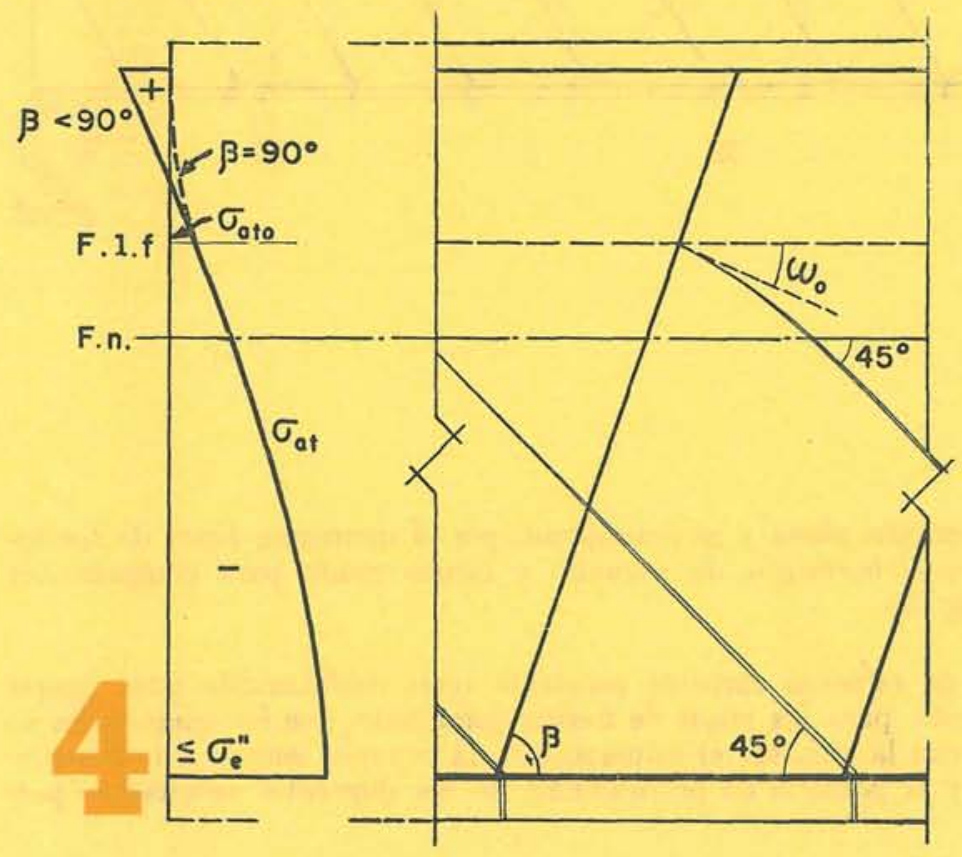

$\sigma_{a t o}=700 \cdot \cos 2\left(\omega_{0}+\beta\right)$

$\mathrm{kg} / \mathrm{cm}^{2}$, siendo $\omega_{o}$ y $\beta$ los ángulos que forman con el eje de la pieza la tangente a la fisura y la dirección de los estribos. A su vez, el ángulo $\omega_{0}$ viene definido por la expresión:

$$
\omega_{0} \simeq \frac{1}{2} \cdot \operatorname{arctg}\left(\frac{2 \cdot \tau_{0}}{\sigma_{x o}}\right)
$$

El valor de la tensión tangencial en esa fibra límite de fisuración tiene por expresión:

$\tau_{o}=\frac{T}{b z}-\cos \beta \cdot q_{a t} \cdot \sigma_{a t o}$

siendo $q_{a t}$ la cuantía geométrica de la armadura transversal por unidad de super- 
ficie horizontal. Esta expresión sustituye a la clásica de la tensión tangencial en la fibra neutra $\left(r_{0}=\frac{T}{b z}\right)$

\section{Tensiones internas en la faja no fisurada}

Partiendo de las leyes admitidas para las tensiones-deformaciones normales horizontales $x$, se plantea el equilibrio del prisma elemental de longitud $d x$ (fig. 5), correspondiente a la faja sin fisurar, teniendo en cuenta las interacciones con las diagonales entre fisuras y los estribos. De esta forma resultan leyes de tensiones internas $\tau$ y $\sigma_{y}$, de expresión complicada, que presentan un aspecto como el de la figura 6 , válidas aproximadamente para los dos casos de fisuras largas o cortas.

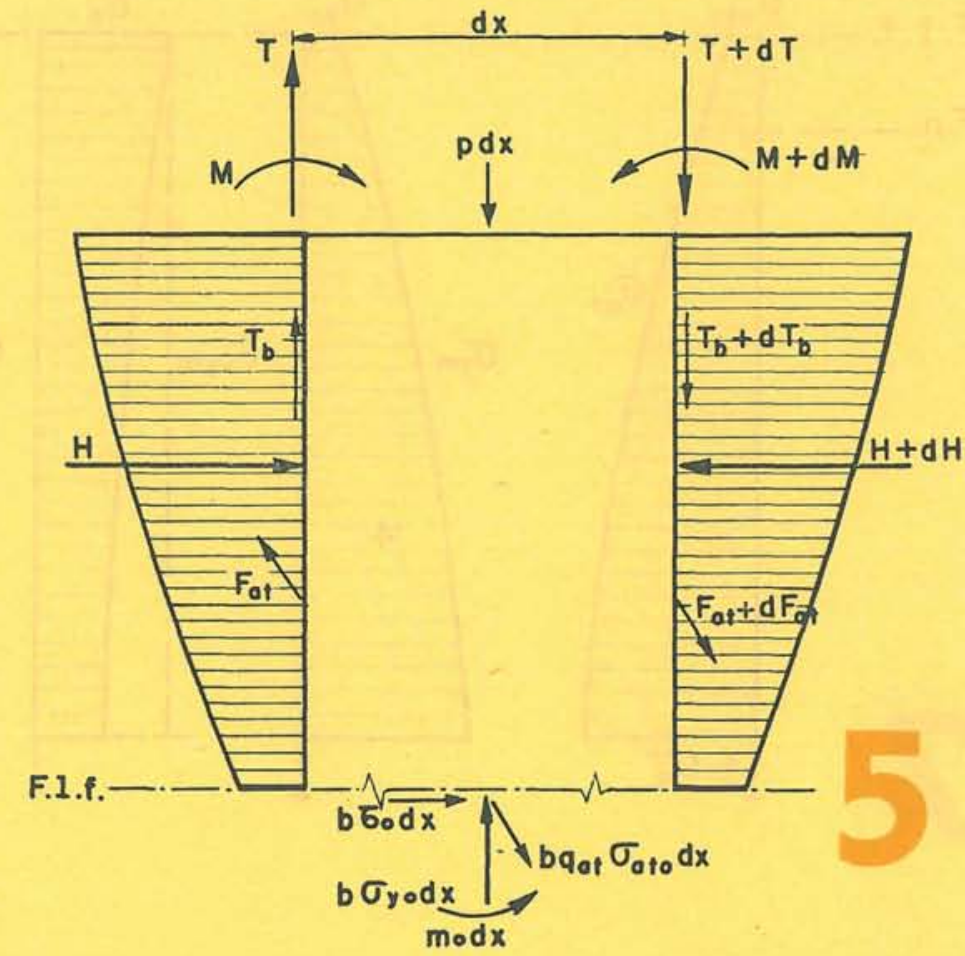

\section{Tensiones eficaces o medias en la faja fisurada}

Aunque se puede perfectamente plantear el equilibrio por una sección mixta que pase por una fisura, hemos preferido plantearlo por una sección recta. A este fin, en la faja fisurada se definen y calculan las que llamaremos «tensiones medias o eficaces», de modo que se equilibren con las tracciones de las armaduras y con las interacciones de la faja no fisurada. Las expresiones de dichas tensiones eficaces son sencillas y se indican a continuación:

$$
\begin{aligned}
\sigma_{y m} & =\operatorname{sen} \beta \cdot q_{a t} \cdot \sigma_{a t} \\
\tau_{x y m} & =\frac{T}{b z}-\cos \beta \cdot q_{a t} \cdot \sigma_{a t} \\
\tau_{y x m} & =\sigma_{y m} \cdot \cot \omega \\
\sigma_{x m} & =\tau_{x y m} \cdot \cot \omega,
\end{aligned}
$$

siendo $\sigma_{a t}$ la tensión en los estribos, y cot $\omega$ la inclinación de la fisura; ambas a la altura de la fibra de que se trata. En la figura 7 se representa el aspecto de estas tensiones medias o eficaces.

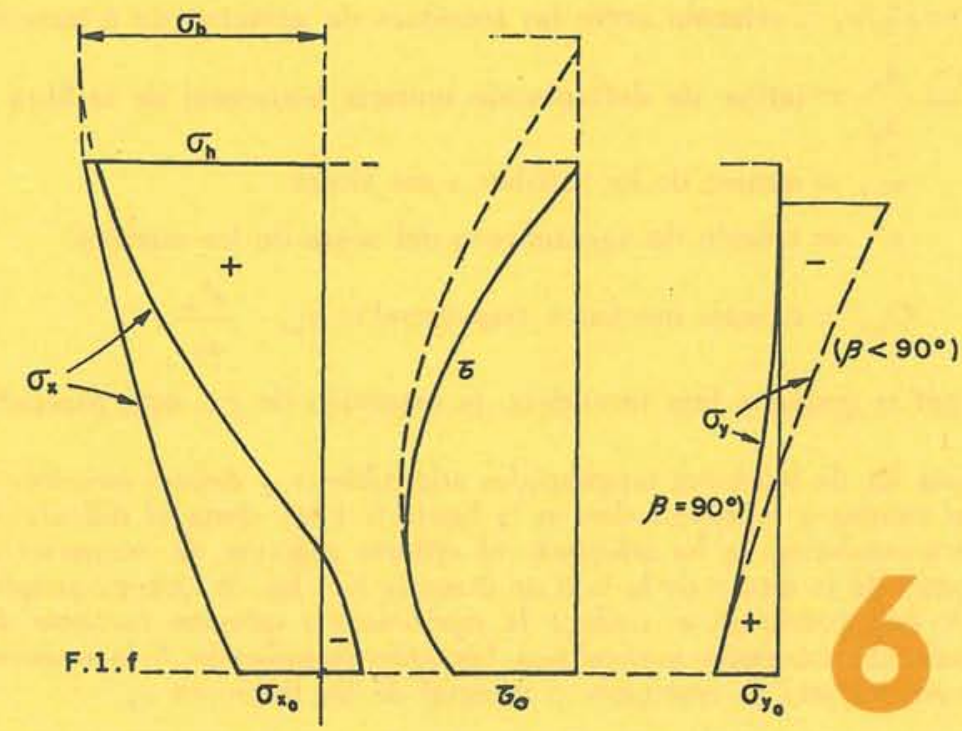




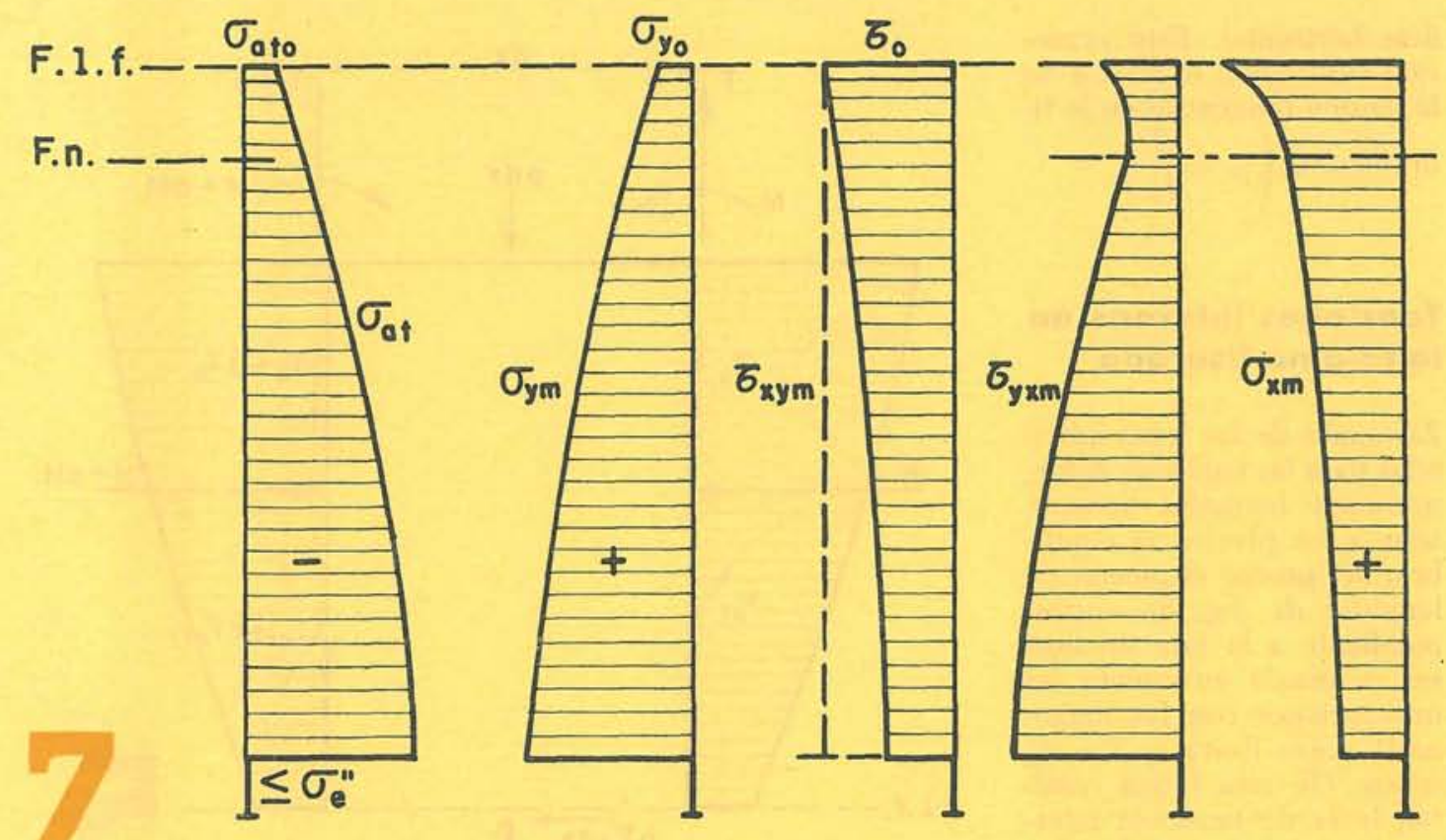

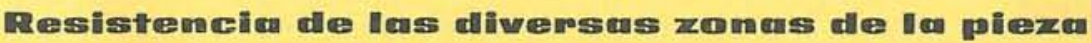

La resistencia del hormigón de la faja no fisurada se plantea partiendo de la resistencia intrínseca del mismo, teniendo en cuenta también las tensiones normales verticales $\sigma_{y}$ que equilibran las tracciones de los estribos (efecto de zunchado). La expresión resultante es:

$$
\frac{\tau_{a d m}}{\sigma_{b}}=\sqrt{\left[r\left(1-\operatorname{sen} \beta \cdot Q_{a t} \cdot \frac{\sigma_{a t}}{\sigma^{\prime \prime}}\right)+(2-v) v\right] \cdot\left[r(1-v)^{2}+\operatorname{sen} \beta \cdot Q_{a t} \cdot \frac{\sigma_{a t}}{\sigma^{\prime \prime}}\right]},
$$

correspondiente a la faja comprimida, siendo:

$r=\sigma_{b}^{\prime} / \sigma_{b}=$ relación entre las tensiones de agotamiento a tracción y a compresión.

$\nu=\frac{\delta_{x}}{\Delta_{b}}$ - índice de deformación unitaria horizontal de la fibra en cuestión.

$\sigma_{a t}=$ tensión de los estribos a esa altura.

$\sigma^{\prime \prime}{ }_{e}=$ tensión de agotamiento del acero de los estribos.

$Q_{a t}=$ cuantía mecánica transversal $=q_{a t} \cdot \frac{\sigma^{\prime \prime}{ }_{e}}{\sigma_{b}}$

Para la pequeña faja extendida, la expresión de $\tau_{a d}$ será parecida.

Esta ley de tensiones tangenciales admisibles $\tau_{a d}$ deberá envolver a la de las tensiones $\tau$ más arriba citadas y representadas en la figura 6 . Pero, dada la dificultad de plantear matemáticamente esta condición se ha adoptado el criterio práctico de compararlas en la fibra media, o sea, a mitad de la altura de la faja no fisurada (ver fig. 8), criterio simple y suficientemente aproximado. De esta condición se deduce la resistencia a esfuerzo cortante de la faja de hormigón no fisurado, de expresión matemática bastante complicada. Y la resistencia de esta faja a las tensiones normales será la resultante o integral de las tensiones $\sigma_{x}$. 
Las resistencias de la faja fisurada al esfuerzo cortante y a las tensiones normales se componen cada una de dos sumandos: uno el debido a las componentes respectivas de las tracciones de las armaduras que atraviesan la sección; el otro, a las resultantes o integrales de las tensiones eficaces $\tau_{y x m}, \sigma_{x m}$ (respectivamente), citadas anteriormente y representadas en la figura 8.

En la zona inmediata al punto de inflexión se justifica que las secciones tienen resistencia superior, a igualdad de cuantía transversal, lo que está, además, de acuerdo con la experiencia.
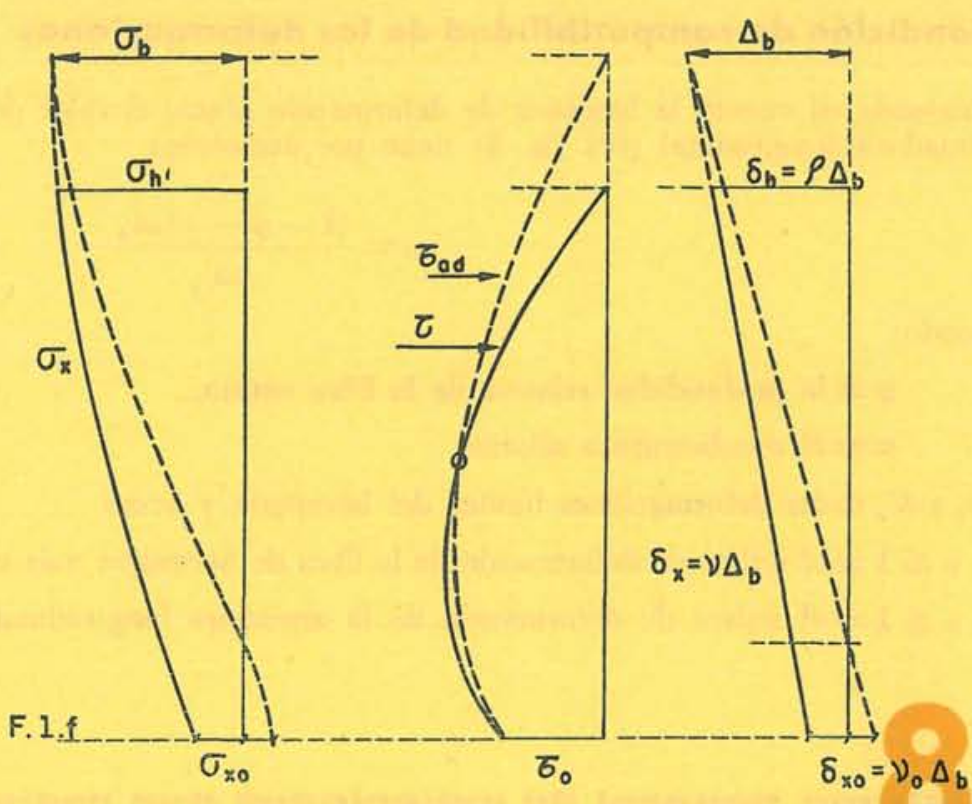

\section{Bom}

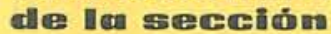

\section{Ecuaciones del equilibrio estático}

Se deducen de igualar las solicitaciones exteriores $N, T, M$, sobre la sección, a las sumas de las resultantes parciales o integrales de las tensiones internas, obteniéndose de esta forma tres inecuaciones, que podrán ser ecuaciones en el caso de agotamiento.

\section{Condición de fisuración en la fibra límite}

A la altura de los extremos superiores de las fisuras (fibra límite de fisuración), la tensión tangencial debe ser igual a la admisible. Así resulta una ecuación que liga al esfuerzo cortante con las demás variables del problema. En valores adimensionales o mecánicos:

$$
t=\frac{T}{b h \sigma_{b}}=\zeta\left[\cos \beta \cdot Q_{a t} \cdot \sigma_{a t o}+\frac{\tau_{o a d m}}{\sigma_{b}}\right]
$$

$\zeta=\frac{z}{h}$, brazo de palanca por unidad de altura.

$Q_{a t}=$ cuantía mecánica transversal, ya citada.

$\tau_{o \text { adm } m}$, se obtiene de hacer $\nu=\nu_{o}=\delta_{x_{o}} / \Delta_{b}$ en la expresión general de $\tau_{a d m}$. 


\section{Condición de compatibilidad de las deformaciones}

Teniendo en cuenta la hipótesis de deformación plana, el valor del índice de deformación de la armadura longitudinal (ver fig. 3) tiene por expresión:

$$
\mu=\frac{(1-\varphi-\alpha)_{\rho} \Delta_{b}}{\varphi \Delta^{\prime}{ }_{e}}
$$

siendo:

$\varphi=$ la profundidad relativa de la fibra neutra.

$\alpha=$ el recubrimiento relativo.

$\Delta_{b}$ y $\Delta_{c}^{\prime}=$ las deformaciones límites del hormigón y acero.

$\rho \leqq 1=$ el índice de deformación de la fibra de hormigón más comprimida.

$\mu \leqq 1=$ el índice de deformación de la armadura longitudinal.

\section{Sistema genemal de ecuaciones que mesuelue el problema}

Así resulta un sistema de cinco ecuaciones, de expresión bastante complicada, que resuelve el problema general. Este sistema puede expresarse como sigue:

Ecuaciones del equilibrio estático:

$$
\begin{gathered}
t=\eta \cdot f_{1}\left(\varphi, \rho, v_{o}, Q_{a t}\right) \\
n=f_{2}\left(\varphi, \rho, v_{o}, \mu, Q^{\prime}, Q_{a t}\right) \\
m+(0,5-\alpha) n=f_{3}\left(\varphi, \rho, v_{o}, Q_{a t}\right)
\end{gathered}
$$

Condición de fisuración en la fibra límite:

$$
t=f_{4}\left(\varphi, \rho, v_{o}, Q_{a t}\right)
$$

Condición de compatibilidad de las deformaciones:

$$
\mu=f_{5}(\varphi, \rho)
$$

siendo:

$t, n, m \quad$ solicitaciones mecánicas o adimensionales.

$Q^{\prime}, Q_{a t} \quad$ cuantías mecánicas longitudinal y tranversal.

$\rho \leqq I$ índices de deformación horizontal de la fibra superior o más comprimida de hor-

$\mu \leqq 1\} \quad$ migón, y de la armadura longitudinal.

$\eta \leqq 1 \quad$ índice de agotamiento transversal.

$v_{o}<\rho \quad$ índice de deformación horizontal en la fibra límite de fisuración.

\section{Estados posibles de agotamiento}

1) Agotamiento transversal simultáneo del hormigón de la faja no fisurada y de las armaduras transversales (incluida la resistencia de las diagonales de hormigón entre fisuras). Dados un 
grupo de valores de $m, n, t, Q$, y haciendo igual a la unidad el índice $\eta$ en la ecuación [1], se obtienen del sistema los de $\varphi, \rho \leqq 1, \mu \leqq 1, v_{o}<\rho, Q_{a t}$ (ver fig. 9).

2) Agotamiento de la armadura longitudinal. Se hace igual a la unidad el índice $\mu$ en las ecuaciones [2] y [5] y se obtienen los valores de $\varphi, \eta \leqq 1, \rho \leqq 1, v_{o}<\rho, Q_{a t}$ (ver fig. 9).

3) Agotamiento de la fibra superior o más comprimida. Se hace igual a la unidad el índice $\rho$ y se obtienen los valores de $\varphi, \eta \leqq 1, \mu \leqq 1, v_{o}<\rho, Q_{a t}$ (ver fig. 9).

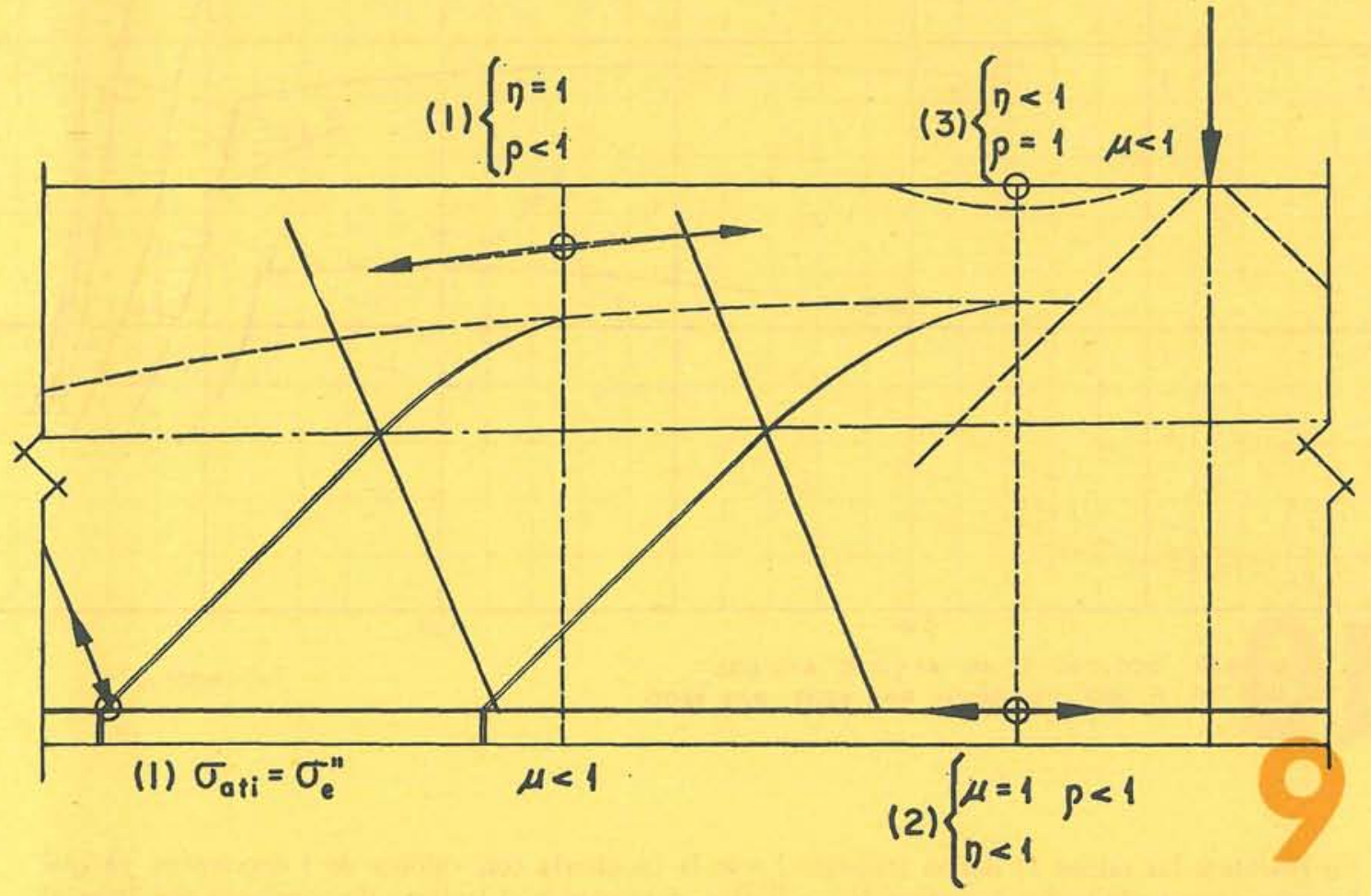

4) Agotamiento superior de la sección. Del estudio de la expresión de la condición de fisuración en la fibra límite, se deduce que al aumentar el valor de $v_{o}$, o sea, al prolongarse las fisuras, va aumentando el valor del esfuerzo cortante mecánico $t$ hasta alcanzar un máximo. Este valor corresponde al concepto de límite superior de la resistencia de la pieza, tal como se ha venido prescribiendo hasta ahora en las instrucciones o normas; la única diferencia es que este límite depende algo de las cuantías de las armaduras, en especial de la transversal.

Para este límite deben ser $\eta, \rho, \mu \leqq 1$.

\section{Resoluchión allel sisstemma}

Dada la complejidad de las ecuaciones y de la interdependencia de algunos de los parámetros, es necesario recurrir al cálculo electrónico para la resolución del sistema. Por tanto, se ha preparado un programa en lenguaje Fortran, con ordenador IBM 1620, obteniéndose los resultados numérica y gráficamente (mediante un «plotter»). En la figura 10 se puede ver, como muestra, un gráfico correspondiente a flexión simple con cuantía longitudinal mecánica de 0,350 y diversas cuantías transversales de estribos a $45^{\circ}$. En este gráfico se distinguen perfectamente las diferentes ramas de curva $1,2,3$ y 4 , que resultan para diferentes cuantías transversales y que corresponden a los respectivos estados de agotamiento anteriormente descritos. 


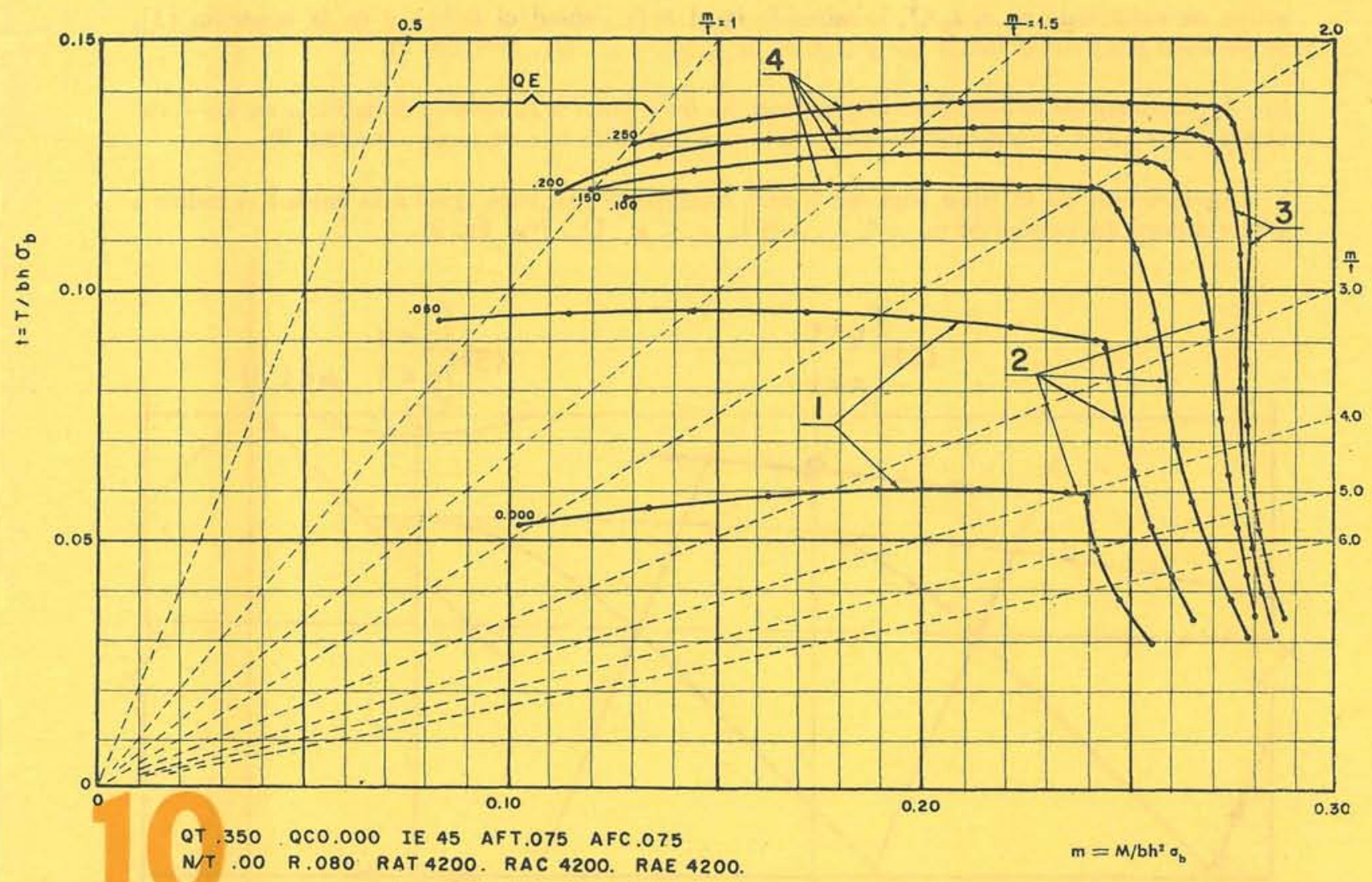

En realidad, las ramas 1) deben terminar hacia la izquierda con valores de $t$ crecientes, ya que las expresiones obtenidas corresponden a flexión dominante; al tratarse de secciones próximas al punto de inflexión, las resistencias al esfuerzo cortante, muy difíciles de obtener teóricamente, son superiores, como ya se ha indicado anteriormente.

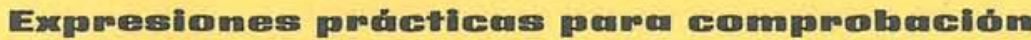

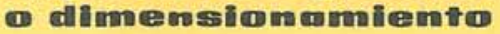

De los resultados obtenidos con el cálculo electrónico, para aceros de 2.400 y $4.200 \mathrm{~kg} / \mathrm{cm}^{2}$ de límite elástico y con valores de $\alpha=0,075$ y $r=\sigma^{\prime}{ }_{b} / \sigma_{b}=0,08$, se han deducido las siguientes expresiones prácticas:

1) Limite de agotamiento transversal.

$$
\begin{aligned}
& 2.400 \mathrm{~kg} / \mathrm{cm}^{2}\left\{\begin{array}{l}
45^{\circ} \\
90^{\circ}
\end{array} t=0,033+0,08 \cdot Q^{\prime}+\left\{\begin{array}{l}
1,6 Q_{a t} \\
0,8 Q_{a t}
\end{array}\right.\right. \\
& 4.200 \mathrm{~kg} / \mathrm{cm}^{2}\left\{\begin{array}{l}
45^{\circ} \\
90^{\circ}
\end{array} t=0,030+0,06 \cdot Q^{\prime}+\left\{\begin{array}{l}
1,6 Q_{a t} \\
0,8 Q_{a t}
\end{array}\right.\right.
\end{aligned}
$$


2) Limite de agotamiento de la armadura longitudinal.

$$
\begin{aligned}
& 2.400 \mathrm{~kg} / \mathrm{cm}^{2}\left\{\begin{array}{l}
45^{\circ} \\
90^{\circ}
\end{array} m=0,030+0,64 Q^{\prime}\left\{\begin{array}{l}
-0,25 t+\left(0,58-0,8 Q^{\prime}\right) Q_{a t} \\
-0,40 t
\end{array}\right.\right. \\
& 4.200 \mathrm{~kg} / \mathrm{cm}^{2}\left\{\begin{array}{l}
45^{\circ} \\
90^{\circ}
\end{array} m=0,030+0,64 Q^{\prime}\left\{\begin{array}{l}
-0,25+\left(0,54-0,8 Q^{\prime}\right) Q_{a t} \\
-0,40 t
\end{array}\right.\right.
\end{aligned}
$$

3) Límite de agotamiento de la fibra superior (sin armadura longitudinal de compresión).

$$
\begin{aligned}
& 2.400 \mathrm{~kg} / \mathrm{cm}^{2}: m=0,310 \\
& 4.200 \mathrm{~kg} / \mathrm{cm}^{2}: m=0,280
\end{aligned}
$$

4) Limite de agotamiento superior de la pieza.

$$
\begin{aligned}
& 2.400 \mathrm{~kg} / \mathrm{cm}^{2}\left\{\begin{array}{l}
45^{\circ}: t=0,118+0,20 Q_{a t}-0,04 Q^{\prime} \\
90^{\circ}: t=0,118+0,24 Q_{a t}-0,04 Q^{\prime}
\end{array}\right. \\
& 4.200 \mathrm{~kg} / \mathrm{cm}^{2}\left\{\begin{array}{l}
45^{\circ}: t=0,122+0,12 Q_{a t}-0,04 Q^{\prime} \\
90^{\circ}: t=0,124+0,16 Q_{a t}-00,4 Q^{\prime}
\end{array}\right.
\end{aligned}
$$

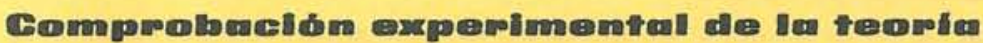

La comprobación experimental es sabido presenta algunas dificultades inherentes a las notables dispersiones en el ensayo de materiales, pequeña escala de las probetas, diferentes definiciones de la rotura, etc. Por ello es necesario realizar amplios programas de ensayos, a ser posible a escala natural.

Nosotros hemos efectuado algunos ensayos con cargo a una beca de la Fundación Juan March, y hemos estudiado algunos resultados de otros ensayos de varios autores, limitándolos a vigas de esbeltez igual o mayor que 6 (curvaturas de 3 ó más). De ellos se deduce que la teoría concuerda sensiblemente con la realidad para una amplia gama de tipos y cuantías de las armaduras.

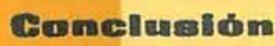

Como consecuencia de todo lo anteriormente expuesto, puede decirse que se trata de un estudio planteado sobre bases rigurosamente teóricas, que aclara gran parte de las incertidumbres sobre el comportamiento del hormigón armado a flexión compuesta y esfuerzo cortante.

No obstante, los autores se proponen continuar su estudio completándolo con las investigaciones que sean necesarias. Por ello, y dado el interés general de esta cuestión, esperan y agradecerán cuantas aportaciones puedan contribuir al progreso de la misma. 


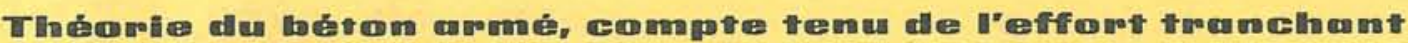

José Antonio López Jamar et José Joaquin Tirado Cruz, Docteurs Ingénieurs des Ponts et Chaussées.

Cet article est un résumé, anticipé de la théorie originale de MM. López Jamar et Tirado Cruz, sur la flexion composée d'éléments de béton armé, compte tenu de l'effort tranchant.

L'élément s'assimile à une poutre en treillis; la tête supérieure, formée par la bande non fissurée, travaille à compression excentrique avec effort tranchant, de même que les diagonales de béton entre fissures. La tête inférieure formée par l'armature lon gitudinale et l'autre série de diagonales ou montants (qui peuvent ne pas exister), travaillent pratiquement à traction pure.

Partant d'une loi parabolique de contraintes normales longitudinales, on obtient rigoureusement celles de contraintes tangentielles les lois de contraintes «efficaces» du béton de la zone fissurée tout au long de la section transversale droite.

Ces lois de contraintes permettent d’étudier les résistances partielles des bandes fissurée et non fissurée, cette dernière au moyen de la résistance intrinsèque. Il en résulte que l'effort tranchant est fonction principalement de la déformation unitaire relative de la fibre ou niveau atteinte par les fissures.

II en résulte quatre formes possibles d'épuisement: transversal simultané du béton et des étriers, armature Iongitudinale, fibre plus comprimée et épuisement supérieur de l'élément. Le système compliqué d'équations qui en résulte est résolu électroniquement et des résultats numériques et graphiques sont obtenus pour les différents cas et armatures possibles, après une verification préalable par essai.

\section{The}

José Antonio López Jamar \& José Joaquín Tirado Cruz, Dr. Civil Engineers.

This article is an advanced summary of the original theory ellaborated by Messrs. Lopez Jamar and Tirado Cruz, on compound bending in reinforced concrete parts, taking account of shear effects.

The authors assume the structure to be similar to an open web girder. The top chord is the uncracked zone, and is assumed to withstand eccentric compression and shear, in the same way as the uncracked diagonal portions of concrete, between cracks. tions (which may no actually exist). These are subject to almost pure tensile loads.

A parabolic distribution of longitudinal stresses is supposed initially, and from this the normal and tangential forces on the non cracked zone is precisely obtained. Also assuming a parabolic stress distribution for the stirrups, the efficient stress dis. tribution laws in the cracked zone of the concrete are obtained.

These stress laws make it possible to study the partial strength of the cracked and uncracked zones: the latter, because of its intrinsic strength. Thus, the shear is mainly dependent on the relative strain of the cracked zone.

There are therefore four possible ways of reaching ultimate failure: simultaneous transversal failure of concrete and stirrups; longitudinal reinforcement failure; compression failure and total failure of the structure. The complex resulting system of equa: reinforcement. A first check of the theory has been made by means of a test.

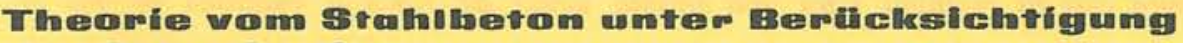

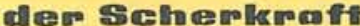

Dr. José Antonio López Jamar und Dr. José Joaquín Tirado Cruz, Strassenbauingenieure.

Dieser Artikel ist eine vorgreifende Zusammenfassung der Theorie von López Jamar und Tirado Cruz über zusammengesetzte Biegung in Spannbetonteilen unter Berücksichtigung der Scherkraft.

Man vergleicht das Stahlbetonteil mit einem Fachwerkträger; der obere Gürtel ohne Risse wird als widerstandsfähig gegen excentrischen Druck und Scherkraft angenommen, ebenso wie die Stahlbetondiagonalen zwischen den Rissen. Der untere Gürtel, der aus der Langsbewehrung und der anderen Gruppe Diagonalen (die nicht zu existieren brauchen) besteht, arbeitet praktisch nur unter reiner Zugkraft.

Nimmt man eine parabolische Verteilung der Längsspannung an, erhält man zwangsläufig die Tangential- und Normalspannung des nicht risshaltigen Gürtels. Desgleichen erhält man die wirksamen Spannungen im risshaltigen Teil entlang des geraden Quer. des nich
schnitts. Diese Spannungsgesetze erlauben es, die Teilwiderstände der risshaltigen und nicht risshaltigen Gürtel zu berechnen, die des letz-
teren mit Hilfe des wahren Widerstandes. Daraus ergibt sich, dass die Scherkraft hauptsächlich eine Funktion der Einheitsdeformation abhängig von der Faser oder dem Niveau der Risse ist.

Daraus ergeben sich 4 verschiedene Erschöpfungszustände des Betons und der Querbewehrung, Erschöpfung der Längsbewehrung, Erschöpfung der ganzen Struktur durch Druck. Das komplizierte Gleichungssystem wird elekttronisch gelöst, wobei man numeri.
sche und graphische Resultate für die verschiedenen Fälle und möglichen Bewehrungen crhält, nachdem man mittels Versuchen einen ersten Nachweis durchgeführt hat. 